\title{
Dose Adjustment of Subcutaneous IgG in Chronic Inflammatory Demyelinating Polyneuropathy
}

\author{
Alanood Alsolaihim Steven K. Baker \\ Neuromuscular Disease Clinic, Divisions of Physical Medicine and Neurology, Department \\ of Medicine, McMaster University, Hamilton, ON, Canada
}

\section{Keywords}

Chronic inflammatory demyelinating polyradiculoneuropathy · Demyelinating polyneuropathy $\cdot$ Subcutaneous Ig $\cdot$ IVIG $\cdot$ Inflammatory polyneuropathy

\section{Abstract}

Chronic inflammatory demyelinating polyradiculoneuropathy (CIDP) is an immune-mediated neuropathy that is characterized by a slowly progressive sensory and motor involvement lasting at least 2 months. We present a CIDP patient on subcutaneous $\mathrm{lg}(\mathrm{SClg})$. Upon fine-tuning his dose from 24 to $28 \mathrm{~g} / \mathrm{week}$, this showed a dramatic improvement in both hand grip (13$25 \%$ ) and dorsiflexion (73-278\%). Follow-up nerve conduction studies also demonstrated significant improvements in latencies, motor amplitudes, and conduction velocities. Ongoing surveillance of CIDP patients receiving SClg therapy is therefore necessary to ensure therapeutic optimization.

\section{Introduction}

Chronic inflammatory demyelinating polyradiculoneuropathy (CIDP) is an immune-mediated neuropathy that is characterized by slowly progressive sensorimotor dysfunction developing over at least 2 months [1]. Diagnosis depends on clinical, laboratory, and electrophysiological criteria [2]. The criteria derived from the European Federation of Neurological 
Societies and Peripheral Nerve Society (EFNS/PNS) are the most widely used criteria, which are based on both clinical and electrodiagnostic abnormalities [2]. Proven treatments for CIDP include immunoglobulins, corticosteroids, and plasmapheresis [3].

In terms of safety profiles of these treatments, IVIg is a preferred long-term treatment. Subcutaneous Ig (SCIg) represents another option for Ig administration, which has been a successful and well-tolerated treatment in immunodeficiency syndromes for more than 25 years [4]. SCIg has been shown to maintain clinical equipoise in stable multifocal motor neuropathy patients who previously received IVIg [5].

\section{Case Report}

We present the case of a 44-year-old male followed up in our neuromuscular clinic at the McMaster University Medical Centre, diagnosed with CIDP 4 years ago based on EFNS/PNS criteria. His disease manifested in the form of weakness and numbness in his distal limbs.

Antibodies against the node of Ranvier proteins were found to be negative.

He responded very well to IVIg $1 \mathrm{~g} / \mathrm{kg}$ every 4 weeks; however, he was complaining of end-of-cycle symptoms manifesting predominantly in reduced grip strength and ankle weakness. Therefore, his treatment was changed to biweekly SCIg at a dose of $24 \mathrm{~g} /$ week, which is equivalent to $96 \mathrm{~g} / \mathrm{month}$ or $1.2 \mathrm{~g} / \mathrm{kg}$.

His grip strength was measured using a JAMAR Dynamometer. His right maximum hand grip strength was $41 \mathrm{~kg}$, while his left was $48 \mathrm{~kg}$. Peak isometric dorsiflexion torque (Biodex) measured $18.4 \mathrm{Nm}$ on the right and $9.2 \mathrm{Nm}$ on the left. His nerve conduction studies showed a prolonged right median distal motor latency of $8.4 \mathrm{~ms}$. His compound muscle action potential (CMAP) amplitude revealed a 65\% conduction block (i.e., $10.1 \mathrm{mV}$ distally and $3.5 \mathrm{mV}$ proximally). Conduction velocity was slowed well into the demyelinating range at $20.5 \mathrm{~m} / \mathrm{s}$, while F-wave latency was prolonged at $52.5 \mathrm{~ms}$. The right common peroneal nerve terminal motor latency (TML) was prolonged at $10.9 \mathrm{~ms}$ with a CMAP amplitude that revealed a $54 \%$ conduction block ( $2.8 \mathrm{mV}$ distally and $1.3 \mathrm{mV}$ proximally). Conduction velocity was slowed to $31.5 \mathrm{~m} / \mathrm{s}$.

Given his persisting clinical symptoms and abnormal electrophysiology, his SCIg dose was augmented to $28 \mathrm{~g} /$ week or $1.4 \mathrm{~g} / \mathrm{kg} /$ month. He reported improvement in his strength 1 month after adjusting his SCIg dose.

At his next follow-up, 6 months later, he reported a significant improvement in his strength. Objective strength testing confirmed the patient-reported outcomes demonstrating significant improvements in both grip (right, $51 \mathrm{~kg}$; left, $54 \mathrm{~kg}$ ) (Fig. 1) and dorsiflexion (right, $31.8 \mathrm{Nm}$; left, $34.8 \mathrm{Nm}$ ) (Fig. 2).

Repeat nerve conduction studies were stable to improved. For example, while the right median TML was unchanged at $8.5 \mathrm{~ms}$, the distal CMAP amplitude increased to $12.6 \mathrm{mV}$ with a dramatic diminution of the conduction block $(12 \%)$ and hastening of conduction velocity at $27.5 \mathrm{~m} / \mathrm{s}$ (34\%). The F-wave latency also improved measuring $41.1 \mathrm{~ms}$ (22\%). The right common peroneal TML shortened to $8.5 \mathrm{~ms}(21 \%)$ despite a stable distal CMAP amplitude of $2.3 \mathrm{mV}$.

Whereas the peroneal conduction velocity improved by only $10 \%$ measuring $34 \mathrm{~m} / \mathrm{s}$, the F-wave latency improved dramatically from 85.6 to $41.1 \mathrm{~ms}$ (52\%). 


\section{Discussion}

This case highlights the critical therapeutic threshold phenomena that beset our CIDP patients and reflects the urgent need to make responsive dosing adjustments when patient-reported outcomes suggest undertreatment. SCIg therapy is ideal in this regard as minor dosing changes can be adjudicated without the complex scheduling challenges of hospital-based periodic infusions. We have collected objective strength data to support the dramatic improvements in both hand grip (13-25\%) and dorsiflexion (73-278\%). Follow-up nerve conduction studies also demonstrated significant improvements in latencies, motor amplitudes, and conduction velocities.

Increased dosing of SCIg compared to IVIg is generally considered to be secondary to lymphatic transport and premature exposure to the antibodies to the reticuloendothelial system - the major source of catabolism. Early predictions suggested a 1:1.5 conversion rate but 1:1 has also been shown to be effective $[4,6]$. Independent of which conversion is initially adopted, future dosing may need to be adjusted to maximize the clinical dose-response relationship.

In conclusion, ongoing surveillance of CIDP patients receiving SCIg therapy is therefore necessary to ensure therapeutic optimization. Furthermore, predictive modelling of IgG dosing in neurologic indications, such as CIDP, fails to recognize the distinct pathophysiologic variability in these patients. Perfunctory IgG dosing then should be replaced with patient-responsive dosing, which is particularly well-suited to home-based SC therapy.

\section{Statement of Ethics}

A written informed consent was obtained from the patient to publish this case report.

\section{Disclosure Statement}

The authors have no conflicts of interest to disclose.

\section{Funding Sources}

The authors received no financial support for the research, authorship, and publication of this article.

\section{Author Contributions}

Steven Baker, MD, McMaster University, Hamilton, ON, Canada: author, design and conceptualization of the study; performed data collection and analysis; drafted, revised and edited the manuscript.

Alanood Alsolaihim, MD, McMaster University, Hamilton, ON, Canada: author; performed data collection and analysis; drafted, revised and edited the manuscript. 


\section{References}

1 Mathey EK, Park SB, Hughes RA, Pollard JD, Armati PJ, Barnett MH, et al. Chronic inflammatory demyelinating polyradiculoneuropathy: from pathology to phenotype. J Neurol Neurosurg Psychiatry. 2015 Sep;86(9):973-85.

2 Van Den Bergh PY, Hadden RD, Bouche P, Cornblath DR, Hahn A; Joint Task Force of the EFNS and the PNS. European federation of neurological societies/peripheral nerve society guideline on management of chronic inflammatory demyelinating polyradiculoneuropathy: report of a joint task force of the European federation of neurological societies and the peripheral nerve society - First revision. J Peripher Nerv Syst. 2010 Mar;15(1):1-9.

3 Oaklander AL, Lunn MP, Hughes RA, Van Schaik IN, Chalk CH. Treatments for chronic inflammatory demyelinating polyradiculoneuropathy (CIDP): an overview of systematic reviews. Cochrane Database Syst Rev. 2017;(1):CD010369.

4 Jolles S, Orange JS, Gardulf A, Stein MR, Shapiro R, Borte M, et al. Current treatment options with immunoglobulin $\mathrm{G}$ for the individualization of care in patients with primary immunodeficiency disease. Clin Exp Immunol. 2015 Feb;179(2):146-60.

5 Katzberg H, Rasutis V, Bril V. Subcutaneous Immunoglobulin (IgPRO20) for Maintenance Treatment in Patients with Multifocal Motor Neuropathy (P7.094). Neurology. 2015;84(14 Supplement):P7.094.

6 Fadeyi M, Tran T. Calculating the dose of subcutaneous immunoglobulin for primary immunodeficiency disease in patients switched from intravenous to subcutaneous immunoglobulin without the use of a doseadjustment coefficient. P T. 2013 Dec;38(12):768-70.

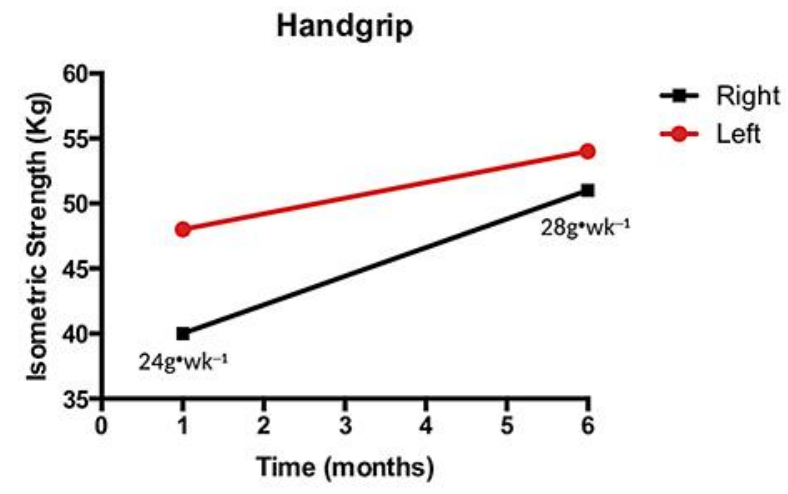

Fig. 1. Hand grip strength improvement after 6 months of fine-tuning SCIg dose from $24 \mathrm{~g} /$ week to 28 g/week. 
(c) 2020 The Author(s). Published by S. Karger AG, Basel

Alsolaihim and Baker: Dose Adjustment of Subcutaneous IgG in Chronic Inflammatory Demyelinating Polyneuropathy

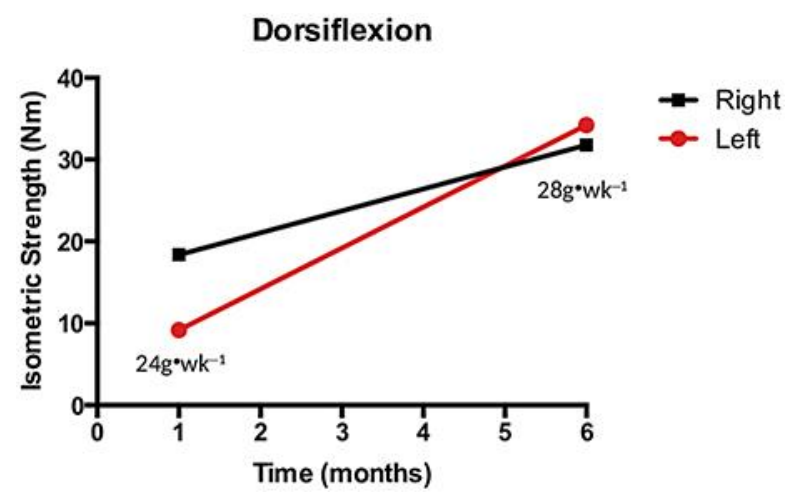

Fig. 2. Dorsiflexion strength improvement after 6 months of fine-tuning SCIg dose from $24 \mathrm{~g} /$ week to 28 g/week. 